\title{
Metabonomic study of the protective effect of Fukeqianjin formula on multi-pathogen induced pelvic inflammatory disease in rats
}

Yan Zhang ${ }^{1 \dagger}$, Wei $\mathrm{Li}^{1 \dagger}$, Liang Zou ${ }^{{ }^{*}}$, Yun Gong ${ }^{2}$, Peng Zhang ${ }^{2}$, Shasha Xing ${ }^{3}$ and Hang Yang ${ }^{1}$

\begin{abstract}
Background: Fukeqianjin formula has been effectively used in the treatment of pelvic inflammatory disease (PID) and the related complications in clinic. Although there have been some studies about the underlying mechanism that focus on its anti-inflammatory and immunoregulatory activities. But the mechanism is still not fully understood. The aim of this study was to investigate the alteration of plasma metabolic profiles in PID rats and the regulatory effect of Fukeqianjin formula on potential biomarkers.

Methods: Pelvic inflammatory model was established by intrauterine inoculation of multiple pathogens combined with mechanical injury of endometrium. Rats were randomly divided into normal group, model group, azithromycin group, high-and low-dose of Fukeqianjin formula treatment group (FF-H, and FF-L, respectively). After 14 days of intragastric administration, the plasm levels of interleukin-1 $\beta$ (IL-1 $\beta$ ) and nitric oxide (NO) were measured. To further recognize and identify potential biomarkers and metabolic pathways, an ultra-performance liquid chromatographyquadrupole-Exactive Orbitrap-mass spectrometry (UPLC-Q-Exactive Orbitrap-MS) metabonomic method combined with multivariate analyses including principal component analysis (PCA), partial least squares discriminant analysis (PLS-DA) and orthogonal partial least squares discriminant analysis (OPLS-DA), was employed to analyze the metabolic profiling.
\end{abstract}

Results: Compared with normal group, the plasma levels of IL-1 $\beta$ and NO were significantly increased in the PID model group $(P<0.05)$, and obviously decreased after high-dose intervention of Fukeqianjin formula $(P<0.01)$. The PCA, PLS-DA and OPLS-DA analysis showed that PID rats were clearly separated from normal rats. Compared with the PID model group, the metabolite profiles of Fukeqianjin formula treatment group was gradually restored to normal. Meanwhile, 14 potential metabolite biomarkers, which were mainly related to the metabolic pathways of intervening glycerophospholipid metabolism, linoleic acid metabolism/alpha-linolenic acid metabolism, amino acid metabolism, arachidonic acid metabolism, and unsaturated fatty acids biosynthesis, have been identified. Fukeqianjin formula exerts good regulatory effect on the abnormal metabolism of PID rats.

Conclusions: Intrauterine inoculation of multiple pathogens combined with mechanical injury of endometrium could significantly disturb the plasma metabolic profiles of rats. Fukeqianjin formula has potential therapeutic effect on multi-pathogen-induced PID by ameliorating metabolism disorders and alleviating the inflammatory response.

Keywords: Fukeqianjin formula, Anti-inflammatory, UPLC-Q-Exactive Orbitrap-MS, Metabonomic, Pelvic inflammatory disease

\footnotetext{
*Correspondence: zouliangcdu@126.com

†Yan Zhang and Wei Li contribute equally as co-first authors

1 School of Medicine, Chengdu University, No. 2025, Cheng Luo Road,

Chengdu 610106, Sichuan, People's Republic of China

Full list of author information is available at the end of the article
} 


\section{Background}

Pelvic inflammatory disease (PID) is a multiple bacterial infection and inflammatory disorder on the upper female genital tract, typically involving the uterus, fallopian tubes, and ovaries [1]. The widespread gynecologic disease, which can lead to serious sequelae, such as tubal infertility, ectopic pregnancy, and chronic pelvic pain, is considered to be a major threat to reproductive age women [2]. Studies based on the updated meta-analysis have also demonstrated that PID might be a potential risk factor of ovarian cancer, especially among Asian women [3]. The most common pathogens cause to PID were Neisseria gonorrhoeae, Chlamydia trachomatis, Mycoplasma genitalium, and gram-negative bacilli [4]. In addition, studies confirm that Staphylococcus aureus, Escherichia coli, and S. sanguinegens were also associated with PID [5, 6]. Currently, PID is commonly treated with broad spectrum antibiotics. A randomized clinical trial confirmed that azithromycin monotherapy or azithromycin monotherapy in combination with metronidazole has an excellent therapeutic effect [7]. Moreover, several antibiotics including ceftriaxone, cefoxitin, doxycycline, and metronidazole also provide a good antibacterial efficacy and high clinical cure rate on pelvic inflammation $[8,9]$.

In recent years, accumulating evidence suggests that Chinese medicine has a remarkable curative effect in the treatment of PID. Research confirms that apart from the infection caused by pathogenic microorganisms, longterm chronic inflammation can also result in the decline of the immune system or pelvic microcirculation disturbance [10], while traditional Chinese medicine has various functions including anti-bacterial, anti-inflammatory, regulating immunity, or promoting blood circulation. An experimental study has demonstrated that Patrinia villosa can provide a good therapeutic effect on pelvic inflammatory rats by significantly reduce the levels of pro-inflammatory cytokines such as IL-6, IL-8 and TNF- $\alpha$ in serum [11]. Studies have shown that Danzhi Decoction not only has a positive anti-inflammatory effect, but also can relieve chronic pelvic pain and ameliorate the pelvic microcirculation disorders in PID mice [12]. The laboratory study confirms that Cortex phellodendri and Humulus japonicus exert good therapeutic effect on mice with chronic PID by inhibiting the expression of inflammatory cytokines and neutrophil infiltration [13]. Furthermore, Feiyangchangweiyan is reported to suppress infiltration and apoptosis of inflammatory cells in uterine tissues by preventing NF- $\mathrm{KB}$ nuclear translocation in pathogen-induced PID rats [14].

Fukeqianjin formula, consisting of Moghania macrophylla, Radix Rosa laevigata, Andrographis paniculata, Mahonia fortunei, Zanthoxylum dissitum hemsl,
Angelica sinensis, Spatholobus suberectus, and Codonopsis pilosula, is a traditional Chinese medicine prescription which has been widely applied to treat various gynecological inflammation disease clinically in China. Previous studies have verified that Fukeqianjin tablet could improve the immune function by promoting the production of IgA, IgG, and IgM in acute PID rats [15]. Furthermore, the experimental study confirmed that the anti-inflammation mechanism of Fukeqianjin tablets might be associated with reducing level of inflammatory cytokines IL-1 $\beta$, IL- 8 and TNF- $\alpha$, and down-regulating of the expression of TNF- $\alpha$, IL-10, and IL-2 mRNA [16, 17]. Additionally, Fukeqianjin tablets can improve the expression of caspases- 3 and caspases-8, mediate inflammatory cells apoptosis, and ultimately alleviate pathological damage of ovarian tissue [18].

As an important branch of systematic biology, metabolomics is mainly used to evaluate the effects of environment, disease status, or drug intervention on endogenous small molecular metabolites like fatty acids, amino acids, peptides, and lipids [19]. Recently, metabolomic investigation has been widely used in the evaluation of biological efficacy and possible mechanism of traditional Chinese medicine. An GC/ MS-based metabolomics research revealed that the metabolic disorders of citric acid circulation, glucose metabolism, unsaturated fatty acids and amino acids in acute liver injury mouse can be regulated by Hedyotis diffusa [20]. A recent metabolomic study suggested that 12 biomarkers were identified in carbon tetrachloride (CCl4)-induced liver fibrosis in rats, and Shu Gan Jian $\mathrm{Pi}$ formula could ameliorate the energy, amino acid, sphingolipid, cytochrome P450, glucose and waterelectrolyte metabolism [21]. Meanwhile, PID-associated metabolomic study in the urine of PID rats based on GC-MS has yet been reported, eighteen potential biomarkers of PID were found, and Patrinia scabiosaefolia Fisch showed a holistic interventional effect on disease-associated metabolomic changes [22, 23].

In the present study, the effect of Fukeqianjin formula on inflammatory cytokines was investigated in PID rats induced by intrauterine inoculation of multiple pathogens combined with mechanical injury of endometrium. Moreover, an UPLC-Q-Exactive Orbitrap-MS-based metabonomic method combined with multivariate analyses including principal component analysis (PCA), partial least squares discriminant analysis (PLS-DA) and orthogonal partial least squares discriminant analysis (OPLS-DA), were used to analyze the metabolic profiling and to recognize and identify potential biomarkers and the metabolic pathways. 


\section{Methods}

Information of experimental design and resources

The information regarding the experimental design, statistics, and resources used in this study are attached in the minimum standards of reporting checklist (Additional file 1).

\section{Chemicals and reagents}

Ferulic acid (Batch No. 110773-200611) was obtained from the National Institutes for Food and Drug Control (Beijing, China). Chlorogenic acid (Batch No. MUST17030620) was obtained from Chengdu Manchester biotech Co., Ltd. (Chengdu, China). Lobetyolin (Batch No. 16022204), Andrographolide (Batch No. 16073103), and dehydrated andrographolide (Batch No. 16022501) were obtained from Chengdu Zhuo Pu Instrument Co., Ltd (Chengdu, China). Fukeqianjin tablets were obtained from Zhuzhou Qianjin pharmaceutical Co., Ltd. (Hunan, China). Azithromycin (Batch No. R37977) was purchased from Pfizer pharmaceutical Co., Ltd. (NY, USA). Staphylococcus aureus (ATCC25923) and E. coli were purchased from Chinese national fungus storehouse. Methanol and acetonitrile with HPLC-grade were purchased from Thermo Fisher Scientific Inc. (Iowa, USA). Formic acid was purchased from Sigma Chemical Co. (St. Louis, MO, USA). Water used in this study was prepared by ULUP Ultrapure Water System (Chengdu, China).

\section{Animals}

Healthy female Sprague-Dawley rats (specific pathogenfree, $220 \pm 20$ g) were supplied by DaShuo Biotechnology Co., Ltd [Approval Number: SCXK (Sichuan) 2015-030, Chengdu, China]. This study was strictly in accordance with the Guidelines for the Care and Use of Laboratory Animals. The animal protocol was approved by the Animal Ethics Committee of Chengdu University.

\section{Preparation and quality control of Fukeqianjin formula}

Fukeqianjin formula powder (4 g) was extracted with $20 \mathrm{~mL} \mathrm{70 \%} \mathrm{ethanol} \mathrm{by} \mathrm{ultrasonic} \mathrm{extraction} \mathrm{for} 60 \mathrm{~min}$. Then the supernatant was concentrated and dissolved with $5 \mathrm{~mL}$ methanol after centrifugation for $10 \mathrm{~min}$ at 12,000 rpm. Samples for High Performance Liquid Chromatography (HPLC) detection were obtained by filtration through a $0.22 \mu \mathrm{m}$ membrane filter.

A HPLC method was established for the identification of the major compounds in Fukeqianjin formula (LC-20AT HPLC system, Shimadzu, Japan). A ZORBAX SB-C18 analytical column $(4.6 \times 250 \mathrm{~mm}, 5 \mu \mathrm{m}$, Agilent, USA) was used with the column temperature maintained at $35{ }^{\circ} \mathrm{C}$. Acetonitrile and $0.1 \%$ phosphoric acid in water were treated as mobile phase A and B, respectively. The gradient elution was programmed as follows: 10\% A-20\%
(0-15 min), 20-47.5\% A (15.1-45 min), 47.5-68.5\% A (45.1-50 min). The detection wavelength is $220 \mathrm{~nm}$. The flow rate was set at $1.0 \mathrm{~mL} / \mathrm{min}$, and the sample injection volume was set at $5 \mu \mathrm{L}$.

\section{Induction of PID model rats}

The preparation of PID model was based on the methods described in the previous literature with some revisions [23]. Rats were anesthetized with $20 \%$ urethane $(5 \mathrm{~mL} / \mathrm{kg}$ ) by intraperitoneal injection. Endometrial tissue was injured by a blunt needle-tip syringe that entered the uterine cavity and pulls back and forth twice along the uterine wall. At the same time, $0.1 \mathrm{~mL}$ of the prepared multi-pathogen solution which was composed of $S$. aureus and $E$. coli $\left(1 \times 10^{8} \mathrm{ccu} / \mathrm{mL}\right.$, respectively $)$ was injected into the ovary, and the rats were positioned upside down for $5 \mathrm{~min}$. Rats in control group were treated identically with saline solution without endometrial tissue injured.

\section{Histopathological analysis of PID model rats}

Ten days after establishment of PID model, the uterine tissue samples of model and normal rats $(n=6$ per group) were randomly collected and fixed in $4 \%$ paraformaldehyde, followed by embedded in paraffin, cut into $5-\mu \mathrm{m}$ sections (RM2235, Leica, Germany). Furthermore, paraffin sections were deparaffinized in xylene, then stained with hematoxylin and eosin (H\&E) and the pathological changes were evaluated randomly, nonconsecutive chosen fields at a magnification of $200 \times$ using optical microscopy (CX21FS1, Olmpus, Japan).

\section{Drug administration and sample collection}

After histological analysis, PID rats were randomly divided into model group, azithromycin group (AZM, $10 \mathrm{mg} / \mathrm{kg}$ ), high-dose of Fukeqianjin formula group (FF$\mathrm{H}, 1.6 \mathrm{~g} / \mathrm{kg}$ ) and low-dose of Fukeqianjin formula group (FF-L, $0.8 \mathrm{~g} / \mathrm{kg}$ ). All drugs were dissolved in distilled water. Normal control group were given equal volume of distilled water. Each group was consisted of 10 rats. After 14 days of intragastric administration, blood samples were collected from abdominal aorta. The plasma samples were centrifuged at $3500 \mathrm{rpm}$ for $10 \mathrm{~min}$ at $4{ }^{\circ} \mathrm{C}$ and immediately stored at $-80{ }^{\circ} \mathrm{C}$ until analysis.

\section{Measurement of inflammatory cytokines}

Concentrations of interleukin-1 $\beta$ (IL-1 $\beta$ ) was measured by enzyme linked immunosorbent assay (ELISA) kits (Batch Number: 2301B61242, MultiSciences Biotech Co., Ltd. Hangzhou, China). The concentration of nitric oxide (NO) was quantified based on the nitric reductase method (Batch Number: 20161114, Nanjing Jiancheng Institute of Biotechnology, Nanjing, China). All date 
was carried out by Varioskan Multifunctional full wavelength microplate reader (Thermo Fisher Scientific, USA) according to the manufacturers.

\section{Plasm sample preparation}

A total of $300 \mu \mathrm{L}$ acetonitrile was added into $100 \mu \mathrm{L}$ of plasm sample for protein precipitation, and then the mixture was vortexed for $3 \mathrm{~min}$, centrifuged at 12,000 rpm for $10 \mathrm{~min}$ at $4{ }^{\circ} \mathrm{C}$. The supernatant was transferred to an auto-sampler vial for UPLC-Q-Exactive Orbitrap-MS analysis.

\section{UPLC-Q-Exactive Orbitrap-MS analysis}

Chromatographic separation was carried out on an Ultimate-3000 RS LC system (Dionex, USA) using an Acquity UPLC BEH C18 column $(2.1 \mathrm{~mm} \times 100 \mathrm{~mm}, 1.7 \mu \mathrm{m}$, Waters, USA) with the column temperature maintained at $35{ }^{\circ} \mathrm{C}$. The mobile phase consisted of $0.1 \%$ formic acid in water (A) and $0.1 \%$ formic acid in acetonitrile (B). The gradient elution was modified as follows: $0-25 \mathrm{~min}$, 5-95\% B; 25-30 min, 95\% B; 30-31 min, 95-5\% B; and 31-35 min, 5\% B. The sample injection volume was set at $10 \mu \mathrm{L}$ for analysis with a flow rate of $0.4 \mathrm{~mL} / \mathrm{min}$.

Mass spectrometry was performed on a Q-Exactive Orbitrap-MS system (Thermo Fisher Scientific, USA) equipped with an electrospray ionization source operating in the positive ion mode with the following parameters: scan type, full MS; scan range, 80 to $1000 \mathrm{~m} / \mathrm{z}$; scan resolution, $7000 \mathrm{~m} / \mathrm{z} / \mathrm{s}$; sheath gas flow rate, $30 \mathrm{arb}$; aux gas flow rate, $10 \mathrm{arb}$; spray voltage, $3.5 \mathrm{kV}$, capillary temperature, $320^{\circ} \mathrm{C}$; aux gas heater temperature, $150{ }^{\circ} \mathrm{C}$.

The quality control (QC) samples mixed by $100 \mu \mathrm{L}$ of 10 experimental samples were determined by duplicate analysis of six injections before analysis to evaluate the precision and repeatability of the instrument. In addition, QC samples were measured every 10 samples during the testing process in order to investigate the stability of analytical method by determining relative standard deviations (RSD) of intensity and retention time of 10 randomly selected characteristic ion peaks of QC samples.

\section{Data analysis}

The raw MS data were exported to MZ mine for normalization treatment before multivariate analysis. The principal component analysis (PCA), partial least-squares discriminant analysis (PLS-DA) and orthogonal partial least-squares discriminant analysis (OPLS-DA) were performed with Metabolomics Univariate and Multivariate Analysis (MUMA) of R software (URL: http://www.Rproject.org/). For potential biomarker identifications, the information was obtained from the following databases: http://www.genome.jp/kegg/, http://metlin.scripps.edu/, http://www.lipidmaps.org/. Potential metabolic pathways were analyzed by Metabo Analyst 2.0. All results were expressed as the mean \pm standard deviation (SD). Oneway analysis of variance (ANOVA) was applied to the statistical analysis and $P$ values less than 0.05 were considered significantly different between groups.

\section{Results}

\section{Quality control of Fukeqianjin formula}

Five major characteristic chemical constituents from Fukeqianjin formula were determined. A typical HPLC chromatogram was shown in Fig. 1. As a result, the concentration of chlorogenic acid, ferulic acid, lobetyolin, andrographolide and dehydrated andrographolide was $0.46 \mathrm{mg} / \mathrm{g}, 0.33 \mathrm{mg} / \mathrm{g}, 0.25 \mathrm{mg} / \mathrm{g}, 2.06 \mathrm{mg} / \mathrm{g}$, and $1.67 \mathrm{mg} / \mathrm{g}$, respectively. Fukeqianjin formula conform to the relevant quality standards.

\section{Histopathological examination of PID model}

In PID group, the uterus of rats were characterized by obvious congestion and edema, epithelial cell proliferation and degeneration, and chronic inflammatory cell infiltration was observed in the endometrium and myometrium. It indicated that the multi-pathogen treatment with endometrial tissue injure could result in the inflammation reaction in the upper genital tract (Fig. 2).

\section{Anti-inflammation activity}

As illustrated in Fig. 3, compared with normal control group, the inflammatory cytokines including IL-1 $\beta$ and NO were significantly increased in PID rats. Treatment with either AZM or FF-H could remarkably decrease the level of IL- $1 \beta$ and NO in plasma, which can be inferred that AZM and FF-H can alleviate genital inflammatory response in rats with pelvic inflammation.

\section{Validation of UPLC-Q-Exactive Orbitrap-MS conditions} The representative UPLC-Q-Exactive Orbitrap-MS total ion chromatogram (TIC) of the plasma samples were shown in Fig. 4. By calculating the peak area, $\mathrm{m} / \mathrm{z}$ data, and retention time of the selected ions, the precision, repeatability of the instrument, and stability of analytical method were evaluated. All of the relative standard deviation (RSD\%) values were less than 6\%, which indicated that this established rat plasma metabolomics analysis method was reliable and accurate.

\section{Pattern recognition and potential biomarkers identification}

First, PCA analysis was used for unsupervised pattern recognition in all groups. Additionally, two supervised pattern recognition methods, PLS-DA and OPLS-DA, were employed to provide better discrimination and to carry out metabolites differences between groups in this 

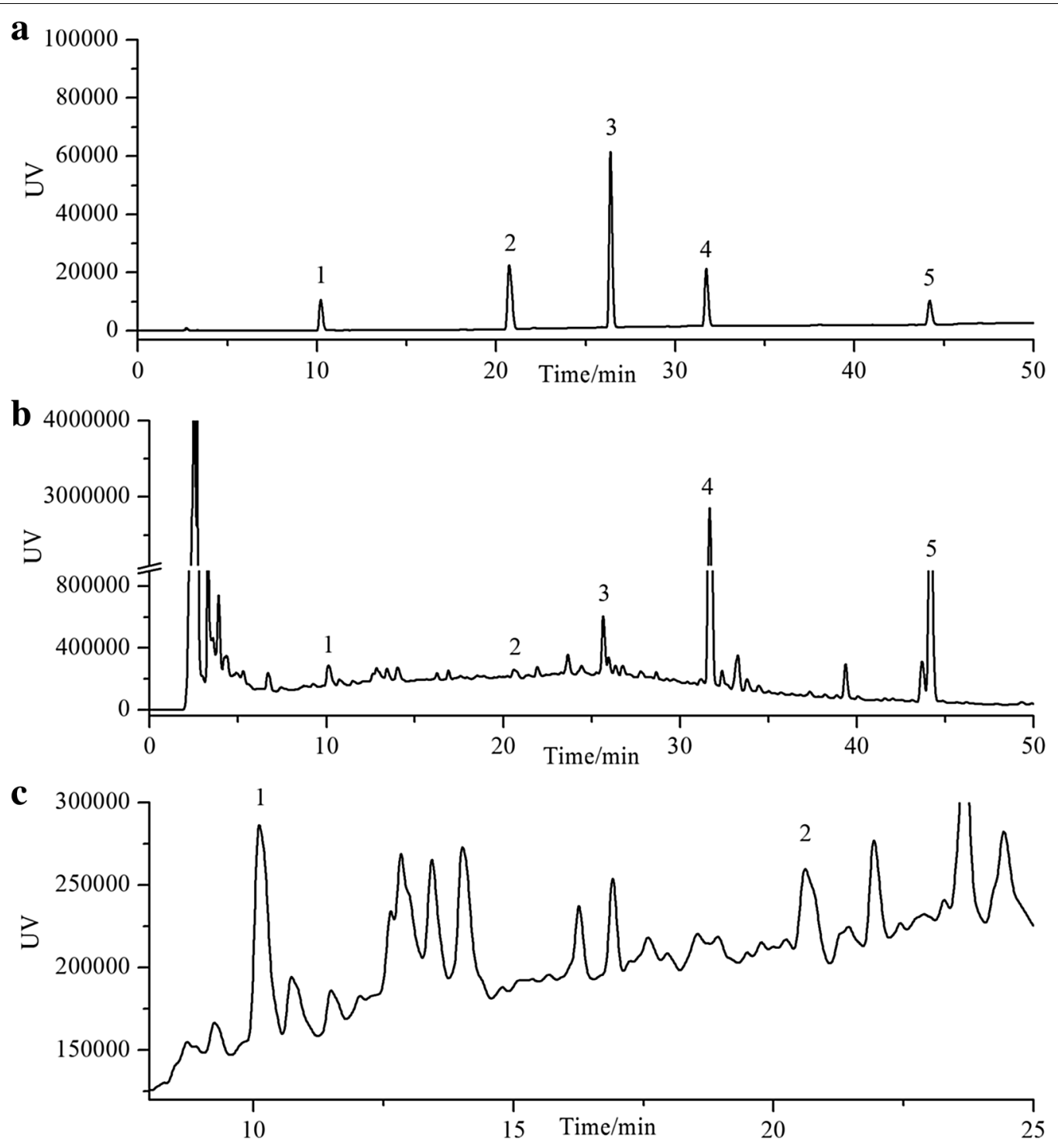

Fig. 1 The typical HPLC chromatogram of Fukegianjin formula. a Mixed standard reference substance. 1: chlorogenic acid (10.163 min); 2: ferulic acid (20.775 min); 3: lobetyolin (26.385 min); 4: andrographolide (31.671 min); 5: dehydrated andrographolide (44.164 min). b Fukeqianjin formula extract (0-50 min). c Fukeqianjin formula extract (8-25 min)

study. As shown in Fig. 5, the corresponding scores plots from PCA, PLS-DA and OPLS-DA showed that PID rats were clearly separated from normal rats, which suggested that intrauterine inoculation of multiple pathogens combined with mechanical injury of endometrium could significantly disturb the plasma metabolic profiles of rats. Meanwhile, compared with the PID model group, the metabolite profiles of Fukeqianjin formula treatment group was gradually restored to normal, indicating that the treatment of Fukeqianjin formula can significantly alleviate the metabolic disorders caused by pelvic inflammation in rats.
A total of 14 potential metabolite biomarkers, including L-alanine, Ser-LysLys-Ile, taurodeoxycholic acid, tridecyl phloretate, 12-ketodeoxycholic acid, diaminomethylidene-L-ornithine, adrenic acid, docosatrienoic acid, Arg-Met-Arg-Thr, Ergine, PC(20:3(8Z,11Z,14Z)/0:0), LysoPC(15:0), LysoPC(16:0), and stearoylcarnitine, which are mainly related to the metabolic pathways of intervening glycerophospholipid metabolism, linoleic acid metabolism/alpha-linolenic acid metabolism, amino acid metabolism, arachidonic acid metabolism, and unsaturated fatty acids biosynthesis were 

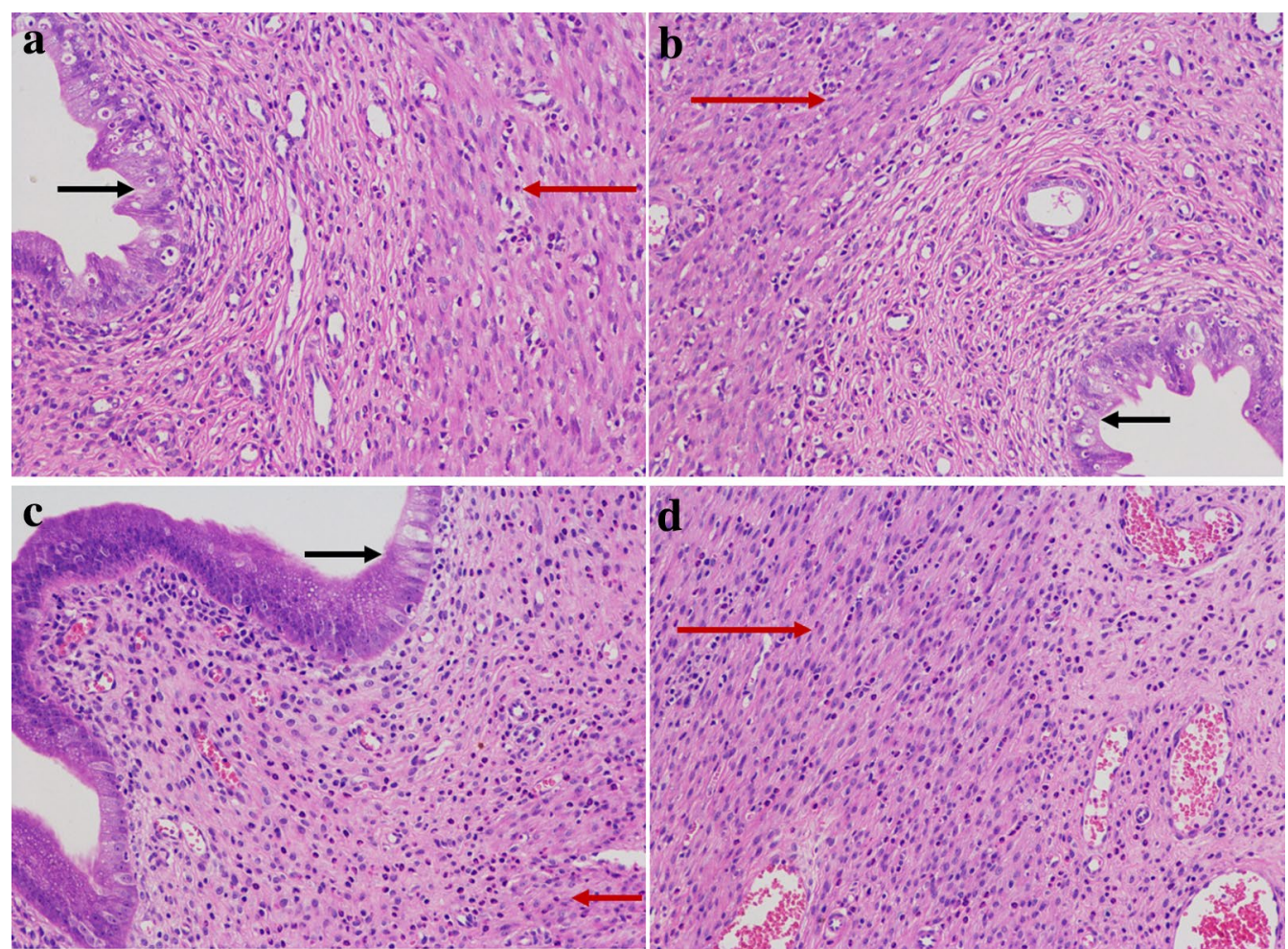

Fig. 2 Representative histopathological micrographs of uterus. a, b Saline solution treatment. c, d Multi-pathogen solution treatment. H\&E stain, $\times 200$. Black arrow stands for endometrium, red arrow stands for myometrium
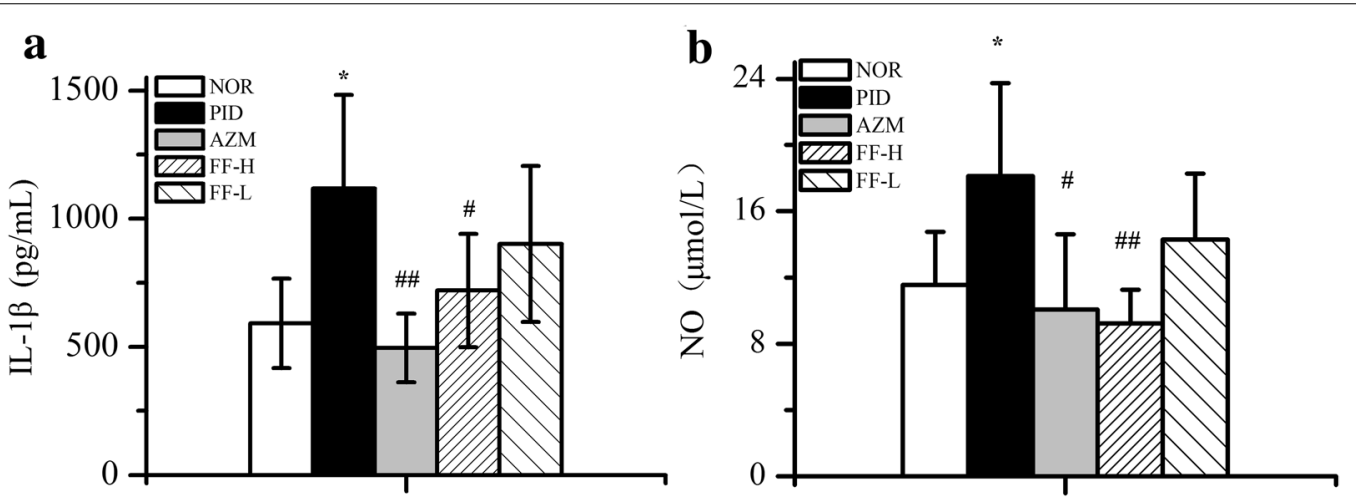

Fig. 3 Effect of Fukeqianjin formula on the inflammatory response in PID rats. a Level of IL-1 $\beta$. $\mathbf{b}$ Level of NO. ${ }^{*} P<0.05$ compared to the control group; ${ }^{\#}<0.05$ and ${ }^{\#} P<0.01$ compared to the PID model group

identified (shown in Table 1). The different intensity of these biomarkers was shown in Fig. 6. The results also suggested that the plasma biomarkers of rats with pelvic inflammation have obvious changes compared with normal rats, and Fukeqianjin formula has potential therapeutic effect on PID rats.

\section{Discussion}

Nitric oxide (NO), an important endogenous bioinformatic molecule produced from L-arginine by constitutive and inducible nitric oxide synthases, plays a key role in inflammatory cascade reaction and immune regulation $[24,25]$. Inhibiting the excessive production of NO 


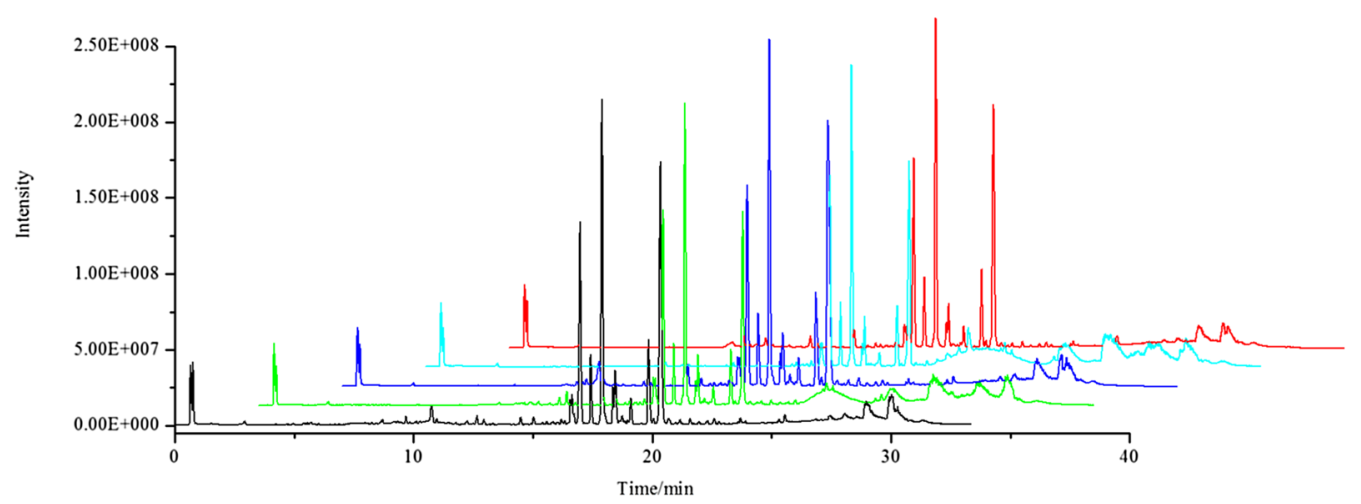

Fig. 4 Representative UPLC-Q-Exactive Orbitrap-MS TIC chromatograms of the plasma samples. Black line: NOR group, Green line: AZM group, Blue line: FF-H group, Light blue line: FF-L group, Red line: PID group

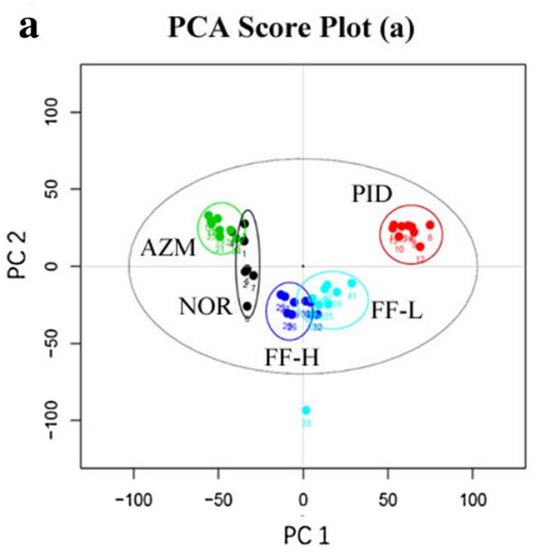

$\mathbf{a}$

c b PLS-DA Score Plot (a)

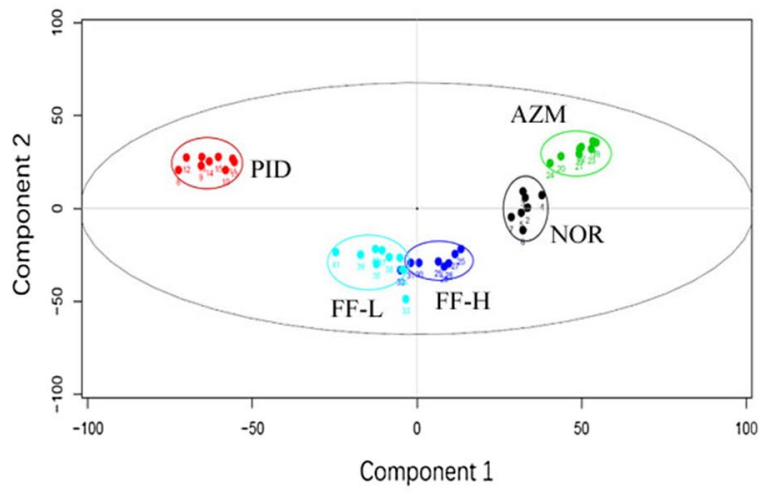

OPLS-DA Score Scatter Plot

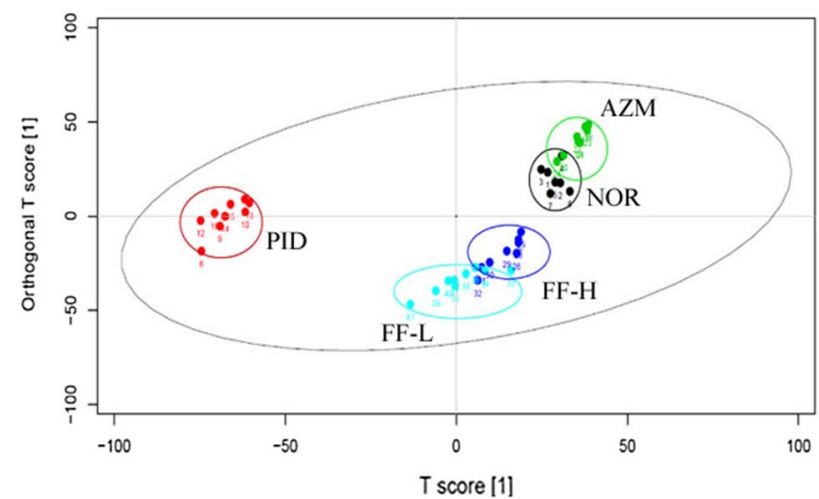

Fig. 5 Scores plots of PCA (a), PLS-DA (b) and OPLS-DA (c) analysis on the PID rat plasm metabolic profiles of normal control (NOR), model (PID), azithromycin treatment (AZM) and Fukeqianjin formula treatment (FF-H, FF-L) group

has been regarded as an effective strategy for the treatment of inflammation-related diseases due to its harmful consequences such as tissue damage in the process of chronic inflammation [26]. It was previously reported that stimulating IL-1 $\beta$ would increase the expression of iNOS, which may further cause an increased production of NO [27]. Study indicated the amount of NO in endometrial tissues was comparatively higher in patients with 
Table 1 The potential metabolite biomarkers

\begin{tabular}{|c|c|c|c|c|c|}
\hline No. & $\mathrm{RT}(\min )$ & Mass $(\mathrm{m} / \mathrm{z})$ & Formula & Identity names & Trend \\
\hline 1 & 0.74 & 90.0544 & $\mathrm{C}_{3} \mathrm{H}_{7} \mathrm{NO}_{2}$ & L-Alanine & $\downarrow$ \\
\hline 2 & 5.67 & 475.3232 & $\mathrm{C}_{21} \mathrm{H}_{42} \mathrm{~N}_{6} \mathrm{O}_{6}$ & Ser-Lys-Lys-Ile & $\downarrow$ \\
\hline 3 & 10.06 & 500.3023 & $\mathrm{C}_{26} \mathrm{H}_{45} \mathrm{NO}_{6} \mathrm{~S}$ & Taurodeoxycholic acid & $\downarrow$ \\
\hline 4 & 11.37 & 357.2775 & $\mathrm{C}_{22} \mathrm{H}_{36} \mathrm{O}_{3}$ & Tridecyl phloretate & $\downarrow$ \\
\hline 5 & 11.39 & 391.2826 & $\mathrm{C}_{24} \mathrm{H}_{38} \mathrm{O}_{4}$ & 12-Ketodeoxycholic acid & $\downarrow$ \\
\hline 6 & 11.54 & 750.3722 & $\mathrm{C}_{28} \mathrm{H}_{51} \mathrm{~N}_{11} \mathrm{O}_{13}$ & Diaminomethylidene-L-ornithine & $\downarrow$ \\
\hline 7 & 12.61 & 355.2618 & $\mathrm{C}_{22} \mathrm{H}_{36} \mathrm{O}_{2}$ & Adrenic acid & $\downarrow$ \\
\hline 8 & 15.34 & 357.2775 & $\mathrm{C}_{22} \mathrm{H}_{38} \mathrm{O}_{2}$ & Docosatrienoic acid & $\downarrow$ \\
\hline 9 & 16.99 & 563.3104 & $\mathrm{C}_{21} \mathrm{H}_{42} \mathrm{~N}_{10} \mathrm{O}_{6} \mathrm{~S}$ & Arg-Met-Arg-Thr & $\downarrow$ \\
\hline 10 & 17.13 & 496.3379 & $\mathrm{C}_{24} \mathrm{H}_{50} \mathrm{NO}_{7} \mathrm{P}$ & LysoPC (16:0) & $\uparrow$ \\
\hline 11 & 17.41 & 268.1477 & $\mathrm{C}_{16} \mathrm{H}_{17} \mathrm{~N}_{3} \mathrm{O}$ & Ergine & $\downarrow$ \\
\hline 12 & 17.78 & 546.3533 & $\mathrm{C}_{28} \mathrm{H}_{52} \mathrm{NO}_{7} \mathrm{P}$ & PC (20:3(8Z,11Z,14Z)/0:0) & $\downarrow$ \\
\hline 13 & 20.18 & 482.3223 & $\mathrm{C}_{23} \mathrm{H}_{48} \mathrm{NO}_{7} \mathrm{P}$ & LysoPC (15:0) & $\downarrow$ \\
\hline 14 & 22.91 & 428.3715 & $\mathrm{C}_{25} \mathrm{H}_{49} \mathrm{NO}_{4}$ & Stearoylcarnitine & $\uparrow$ \\
\hline
\end{tabular}

endometriosis [28]. Rocha et al. reported that NO levels appeared to be elevated in women with chronic pelvic pain diagnosed as secondary stage to endometriosis, and was directly associated with reduction in pain intensity and increase in pain threshold after treatment [29]. Meanwhile, recent experiments have shown that the productions of inflammatory cytokines including IL-1 $\beta$, IL-6, and TNF- $\alpha$ were significantly increased in upper genital tract of PID rats $[16,17]$. In patients with upper genital tract inflammatory diseases, the level of interleukins IL-1 $\beta$ were prominently elevated [30]. In this study, NO and IL-1 $\beta$ level in PID rat plasma was detected to evaluate the anti-inflammatory function of Fukeqianjin formula. Results show that the level of NO and IL-1 $\beta$ were significantly increased (Fig. 3), indicating inflammatory response has been induced in PID rats. Moreover, treatment with FF-H remarkably decrease these cytokines levels, suggesting a potent anti-inflammatory effect of FF-H.

A systematic review and meta-analysis drew a conclusion that $A$. paniculata, an important drug in Fukeqianjin formula, was safe and effective in the treatment of acute respiratory tract infections [31]. Andrographolide is the main chemical constituent of A. paniculata and is also considered to be a major contributor to the therapeutic activity [32, 33]. It has been reported that andrographolide has a broad-spectrum antimicrobial activity against both Gram (+) and Gram (-), especially to $S$. aureus, Bacillus subtilis, E. coli, Pseudomonas aeruginosa, and Klebsiella pneumoniae [34]. In addition, andrographolide was able to inhibit Chlamydia trachomatis-induced human cervical epithelial cell infection and reduce the level of inflammatory factors such as IFN $\gamma$-induced protein10, CXCL8, and IL-6 [35]. Study also confirmed that andrographolide administration in both LPS-activated RAW264.7 cells and peritoneal macrophages could decrease IFN- $\beta$, iNOS, TNF- $\alpha$, and COX-2 expression as well as the downstream NO and PGE2 productions [36]. Andrographis paniculata has also been proved to have potent therapeutic effect on pathogen-induced PID rats by significantly reduce the excessive secretion of cytokines and chemokines including IL-1 $\beta$, IL-6, CXCL-1, and MCP-1 through blocking the NF- $\mathrm{KB}$ signal pathway transduction [37]. Based on our experimental results and previous studies, we speculate that $A$. paniculata may be one of the main anti-inflammatory drugs in Fukeqianjin formula.

Besides, it was previously reported that the total flavonoids from $R$. laevigata Michx fruit markedly downregulated the expression levels of IL-1 $\beta$, IL-6, TNF- $\alpha$ by inhibiting NF- $\mathrm{kB}$ and AP-1 transcriptional activities during inflammatory courses, and thus has protective effect on pathological processes including renal and hepatic ischemia-reperfusion damage [38, 39]. Berberine, as the main ingredient of Mahonia fortunei, was widely recognized for its considerable anti-inflammatory effects. Study has demonstrated that berberine has a protective effect on adenomyosis, a complication of PID, by inhibiting the expression of IL-6, IL-8, TGF- $\beta$, VEGF and MMP-2 [40]. Its anti-inflammatory mechanism mainly involves signaling pathways such as NF- $\mathrm{K}$ $B$, AMPK, and caspase-1/IL-1 $\beta$ inflammatory signal transduction axis $[41,42]$. Combination of the above herbal medicine can enhance the anti-inflammatory effect of the compound, and produce a good therapeutic effect on pelvic inflammation. 


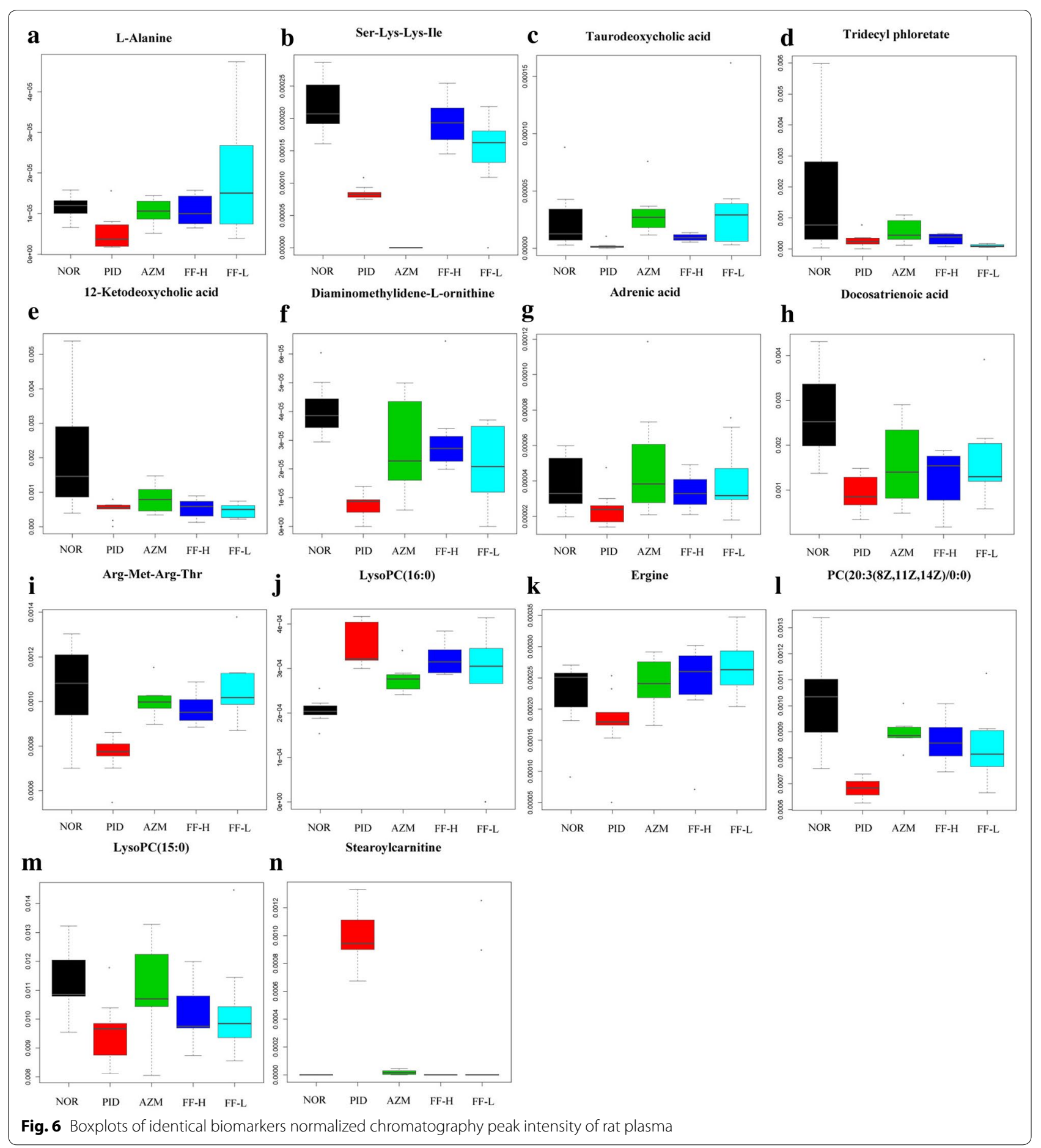

At the same time, although PID is recognized as a multiple bacterial infectious disease, chronic inflammation and correlative complications also participate in its progression. So, in addition to short-term antimicrobial targets, long-term sequelae prevention and treatment is also an important therapeutic goal [43]. Some studies indicates that chronic PID are often under a situation of microcirculation disorder and immune deficiency. Angelica sinensis is commonly used in Chinese medicine to promote hematopoietic function, improve microcirculation and regulate the immune system [44]. Ferulic acid, Z-ligustilide and polysaccharides are the main chemical 
constituents of Angelica sinensis [45]. Liu et al. reported polysaccharides may promote the recovery of platelets and other blood cells and the formation of hematopoiesis in rat by regulating the PI3K/Akt pathway [46]. Spatholobus suberectus contains various types of polyphenolic compounds mainly including flavonoids, isoflavones, flavonols, and flavanones [47]. Among them, total flavonoids of $S$. suberectus have been proved to inhibit oxidative stress and regulate immune function, and it can also play an anti-viral role [48]. Through reasonable compatibility, the whole compound can not only achieve good anti-inflammatory effect, but also improve microcirculation and immunity. It has significant advantages in the treatment of pelvic inflammatory disease and its complications.

In addition, since the complex composition of Chinese herbal compound, it is difficult to elucidate its mechanism of action. Metabonomics methodology covers a wide range of substances, focusing on holistic and dynamic evaluation, which was in coincidence with the holistic theory of traditional Chinese medicine. In recent years, metabonomics has been widely used in the study of inflammatory diseases. Previous studies have shown that collagen-induced arthritis was characterized by metabolic disorders of lipid, tricarboxylic acid cycle, tryptophan and phenylalanine metabolism [49]. According to Ahn et al. the mechanism of curcumin in the treatment of rheumatoid arthritis may be related to the abnormal regulation of glycine, citrulline, arachidonic acid and saturated fatty acid levels [50]. In this study, the plasma metabolomics method based on UPLC-Q-Exactive Orbitrap-MS was used to investigate the underlying mechanism of Fukeqianjin formula on pelvic inflammation. Our results demonstrate that 14 endogenous compounds can be considered as potential metabolite biomarkers of PID (Table 1). Studies have confirmed that the metabolites including amino acids, fatty acids, organic acids, and sugars in urine could considered to be the potential biomarkers of PID [22]. In addition, Patrinia scabiosaefolia Fisch is believed to play a therapeutic role in pelvic inflammation by regulating tricarboxylic acid circulation, glucose metabolism, and amino acid metabolism [23]. However, the above studies were based on the urine metabolomics of gas chromatography-mass spectrometry and our study can be used as complementary biological information for PID.

Previous studies suggested that the lipids metabolism, including bile acids and unsaturated fatty acids, is involved in the occurrence and development of inflammatory reaction [51]. Taurine deoxycholic acid has been shown to ameliorate insulin resistance in mice by inhibiting the increase of phospholipid, myelin sheath and neuramide [52]. Moreover, taurine deoxycholic acid can significantly up-regulate the expression of MUC4 (a membrane-bound mucin) by activating phosphatidylinositol 3-kinase, thereby affecting cell proliferation and tumor progression [53, 54]. As a long chain polyunsaturated fatty acids, docosatrienoic acid is of great benefit to health. As a metabolite of arachidonic acid, adrenic acid can be converted into a variety of oxygenated metabolites, and plays an important role in cardiovascular system and immune system [55]. In this study, a variety of bile acids and unsaturated fatty acids, including taurodeoxycholic acid, 12-ketodeoxycholic acid, docosatrienoic acid and adrenic acid, were identified (Table 1). After treatment with Fukeqianjin formula for 14 days can significantly correct the abnormal changes of these substances (Fig. 6).

Studies have shown that some kinds of phosphatidylcholine (PC) were important biomarkers of inflammation. Glycerophospholipids metabolism profiles can characterize the progress of inflammation and provide valuable evidence for the diagnosis and prognosis of inflammation [56]. It has been confirmed that various drugs in Fukeqianjin formula can affect lipid metabolism. For instance, the total flavonoids of $R$. laevigata Michx can regulate lipid metabolism in LPS-induced liver injury mice by mainly reducing the expression levels of fatty acid synthase, acetyl coenzyme A carboxylase-1, and stearoyl coenzyme A desaturase- 1 , and improving the level of carnitine palmitoyltransferase 1 [57]. Besides, some new polyynes from Codonopsis pilosula may affect lipid metabolism by inhibiting the expression of squalene monooxygenase gene in HepG2 cells [58]. Our data suggested that there were significant changes in biomarkers, including LysoPC (16:0), PC (20:3(8Z,11Z,14Z)/0:0), and LysoPC (15:0), in PID rats, which could be regulated by Fukeqianjin formula intervention (Fig. 6).

Amino acids are not only essential substances of proteins, but also as signal molecules regulate various physiological functions of the organism, such as precursors of many neurotransmitters and hormones, and intermediates of TCA circulation and glyconeogenesis [59]. Previous reports have demonstrated that branched-chain amino acids can increase the expression of eNOS and nitrotyrosine, and induce inflammatory response by activating the transcription factor NF- $\mathrm{kB}$ in endothelial cells [60]. Four potential biomarkers related to amino acid metabolism, including L-alanine, Ser-Lys-Lys-Ile, diaminomethylidene-L-ornithine, and Arg-Met-Arg-Thr, have been shown in Table 1. Fukeqianjin formula was observed to have an obvious regulatory effect on the abnormal changes of these substances (Fig. 6). To summarize, the results illustrated that the metabolic pathways were mainly related to glycerophospholipid metabolism, linoleic acid metabolism/alpha-linolenic acid metabolism, 
amino acid metabolism, arachidonic acid metabolism, and unsaturated fatty acids biosynthesis. Fukeqianjin formula has potential therapeutic effect on multi-pathogeninduced PID rats by ameliorating metabolism disorders.

\section{Conclusions}

In summary, these results indicate that intrauterine inoculation of multiple pathogens combined with mechanical injury of endometrium could significantly disturb the plasma metabolic profiles of rats. Fukeqianjin formula has potential therapeutic effect on multi-pathogeninduced PID rats by ameliorating metabolism disorders and alleviating the inflammatory response.

\section{Additional file}

Additional file 1. The minimum standards of reporting checklist.

\begin{abstract}
Abbreviations
PID: pelvic inflammatory disease; FF: Fukeqianjin formula; AZM: azithromycin; IL-1 13: interleukin-1 B; IL-8: interleukin-8; IL-10: interleukin-10; IL-2: interleukin-2; NO: nitric oxide; TNF-a: tumor necrosis factor-a; NF-kB: nuclear factor-kb; IgA: immunoglobulin A; IgG: immunoglobulin G; IgM: immunoglobulin M; UPLC-Q-Exactive Orbitrap-MS: ultra-performance liquid chromatographyquadrupole-Exactive Orbitrap-mass spectrometry; NMR: nuclear magnetic resonance; GC-MS: gas chromatography-mass spectrometer; HPLC: high performance liquid chromatography; PCA: principal component analysis; PLS-DA: partial least squares discriminant analysis; OPLS-DA: orthogonal partial least squares discriminant analysis; CCl4: carbon tetrachloride; H\&E: hematoxylin and eosin; QC: quality control; RSD: relative standard deviations; MUMA: Metabolomics Univariate and Multivariate Analysis; SD: standard deviation; ANOVA: one-way analysis of variance; TIC: total ion chromatogram;

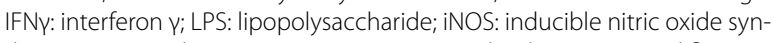
thase; COX-2: cyclooxygenase-2; PGE2: prostaglandin E2; TFs: total flavonoids; VEGF: vascular endothelial growth factor; TGF- $\beta$ : transforming growth factor- $\beta$; AMPK: adenosine $5^{\prime}$-monophosphate (AMP)-activated protein kinase; MMP-2: matrix metalloproteinases- 2 .
\end{abstract}

\section{Authors' contributions}

$L Z$ and $Y G$ designed the research. $Y Z, W L, H Y$ and $P Z$ performed this study. WL, YZ and SSX analyzed the data. YZ and WL wrote the paper. YZ was responsible for the critical revision of the paper. All authors read and approved the final manuscript.

\section{Author details}

'School of Medicine, Chengdu University, No. 2025, Cheng Luo Road, Chengdu 610106, Sichuan, People's Republic of China. ${ }^{2}$ Zhuzhou Qianjin Pharmaceutical Ltd. Co., No. 801 Zhuzhou Avenue, Tianyuan District, Zhuzhou 412000, Hunan, People's Republic of China. ${ }^{3}$ Drug Clinical Trial Center, Affiliated Hospital of Chengdu University, 2nd Ring Road, Jinniu District, Chengdu 610081, Sichuan, People's Republic of China.

\section{Acknowledgements}

Not applicable.

\section{Competing interests}

The authors declare that they have no competing interests.

Availability of data and materials

Please contact author for data requests.

\section{Consent for publication}

All authors have provided consent for publication in the journal of Chinese Medicine.

\section{Ethics approval and consent to participate}

Institutional animal ethics committee approval for the experimental protocol was obtained from Animal Ethics Committee Guidelines of Chengdu University (Chengdu, China) before initiation of the study.

\section{Funding}

This work was supported by Chengdu science and Technology Bureau (2016-HM01-00096-SF), Sichuan Provincial Department of Education (17TD0010), Health and Family Planning Commission of Chengdu-Key disciplines of clinical pharmacy.

\section{Publisher's Note}

Springer Nature remains neutral with regard to jurisdictional claims in published maps and institutional affiliations.

Received: 18 September 2018 Accepted: 29 November 2018 Published online: 10 December 2018

\section{References}

1. Mitchell C, Prabhu M. Pelvic inflammatory disease: current concepts in pathogenesis, diagnosis and treatment. Infect Dis Clin N Am. 2013;27(4):793-809.

2. Haggerty CL, Schulz R, Ness RB. Lower quality of life among women with chronic pelvic pain after pelvic inflammatory disease. Obstet Gynecol. 2003;102(5 Pt 1):934-9.

3. Zhou Z, Zeng F, Yuan J, Tang J, Colditz GA, Tworoger SS, et al. Pelvic inflammatory disease and the risk of ovarian cancer: a meta-analysis. Cancer Causes Control. 2017;28(5):415-28.

4. Sharma H, Tal R, Clark NA, Segars JH. Microbiota and pelvic inflammatory disease. Semin Reprod Med. 2014;32(1):43-9.

5. Haggerty CL, Totten PA, Tang G, Astete SG, Ferris MJ, Norori J, et al. Identification of novel microbes associated with pelvic inflammatory disease and infertility. Sex Transm Infect. 2016;92(6):441-6.

6. Spencer TH, Umeh PO, Irokanulo E, Baba MM, Spencer BB, Umar Al, et al. Bacterial isolates associated with pelvic inflammatory disease among female patients attending some hospitals in Abuja, Nigeria. Afr J Infect Dis. 2014;8(1):9-13.

7. Bevan $C D$, Ridgway $G L$, Rothermel CD. Efficacy and safety of azithromycin as monotherapy or combined with metronidazole compared with two standard multidrug regimens for the treatment of acute pelvic inflammatory disease. J Int Med Res. 2003;31(1):45-54.

8. Piyadigamage A, Wilson J. Improvement in the clinical cure rate of outpatient management of pelvic inflammatory disease following a change in therapy. Sex Transm Infect. 2005;81(3):233-5.

9. Ness RB, Soper DE, Holley RL, Peipert J, Randall H, Sweet RL, et al. Effectiveness of inpatient and outpatient treatment strategies for women with pelvic inflammatory disease: results from the pelvic inflammatory disease evaluation and clinical health (peach) randomized trial. Am J Obstet Gynecol. 2002;186(5):929-37.

10. Zhou J, Qu F. Treating gynaecological disorders with traditional Chinese medicine: a review. Afr J Tradit Complement Altern Med. 2009;6(4):494-517.

11. Zheng Y, Jin Y, Zhu HB, Xu ST, Xia YX, Huang Y. The anti-inflammatory and anti-nociceptive activities of Patrinia villosa and its mechanism on the proinflammatory cytokines of rats with pelvic inflammation. Afr J Tradit Complement Altern Med. 2012;9(3):295-302.

12. Bu X, Liu Y, Lu Q, Jin Z. Effects of "danzhi decoction" on chronic pelvic pain, hemodynamics, and proinflammatory factors in the murine model of sequelae of pelvic inflammatory disease. Evid Based Complement Altern Med. 2015;2015:547251.

13. Oh Y, Kwon YS, Jung BD. Anti-inflammatory effects of the natural compounds cortex Phellodendri and Humulus japonicus on pelvic inflammatory disease in mice. Int J Med Sci. 2017;14(8):729-34. 
14. Li Y, Liu Y, Yang Q, Shi Z, Xie Y, Wang S. Anti-inflammatory effect of Feiyangchangweiyan capsule on rat pelvic inflammatory disease through JNK/NF-KB pathway. Evid Based Complement Altern Med. 2018;2018:8476147.

15. Yuan J, Guo J, Wu C, Yue Z, Zeng G. Effect of Qianjin tablets on serum $\lg A, \lg G$ and $\lg M$ in acute pelvic inflammation in rats. J TCM Univ Hunan. 2010;30(9):87-9 (In Chinese).

16. Lu Y, Qu J, Guo J, Zuo Z, Shi Z, Wang X, Gui L. Effect of Fuke Qianjin tablet on TNF-a and IL-2 mRNA transcription in the uteri and ovary tissues of acute pelvic inflammatory disease in rats. Chin Tradit Patent Med. 2012;34(1):29-33 (In Chinese)

17. Li X, Guo J, Shi Z, Nie J. Effect of Fuke Qianjin tablets on inflammatory cytokines in blood serum in rats with chronic pelvic inflammatory disease. Chin J Exp Tradit Med Formulae. 2013;19(10):226-8 (In Chinese).

18. Nie J, Li X, Shi Z, Guo J. Influence of FukeQianjin tablets to rat's caspase-3 and caspase-8 expression of ovary with chronic pelvic inflammatory disease. Northwest Pharm J. 2014;29(4):385-7 (In Chinese)

19. Wang X, Sun H, Zhang A, Sun W, Wang P, Wang Z. Potential role of metabolomics apporoaches in the area of traditional Chinese medicine: as pillars of the bridge between Chinese and Western medicine. J Pharm Biomed Anal. 2011;55(5):859-68.

20. Dai M, Wang F, Zou Z, Xiao G, Chen H, Yang H. Metabolic regulations of a decoction of Hedyotis diffusa in acute liver injury of mouse models. Chin Med. 2017;12:35.

21. Jiang H, Qin XJ, Li WP, Ma R, Wang T, Li ZQ. Effects of Shu Gan Jian Pi formula on rats with carbon tetrachloride-induced liver fibrosis using serum metabonomics based on gas chromatography-time of flight mass spectrometry. Mol Med Rep. 2017;16(4):3901-9.

22. Zou W, Wen X, Sheng X, Zheng YI, Xiao Z, Luo J, et al. Gas chromatography-mass spectrometric method-based urine metabolomic profile of rats with pelvic inflammatory disease. Exp Ther Med. 2016;11(5):1653-60.

23. Zou W, Wen X, Zheng Y, Xiao Z, Luo J, Chen S, et al. Metabolomic study on the preventive effect of Patrinia scabiosaefolia Fisch on multipathogen induced pelvic inflammatory disease in rats. Evid Based Complement Altern Med. 2015;2015:170792.

24. Niedbala W, Alves-Filho JC, Fukada SY, Vieira SM, Mitani A, Sonego F, et al. Regulation of type 17 helper T-cell function by nitric oxide during inflammation. Proc Natl Acad Sci USA. 2011;108(22):9220-5.

25. Xu L, Zhu J, Yin W, Ding X. Astaxanthin improves cognitive deficits from oxidative stress, nitric oxide synthase and inflammation through upregulation of PI3K Akt in diabetes rat. Int J Clin Exp Pathol. 2015;8(6):6083-94.

26. Zhou MX, Wei X, Li AL, Wang AM, Lu LZ, Yang Y, et al. Screening of traditional Chinese medicines with therapeutic potential on chronic obstructive pulmonary disease through inhibiting oxidative stress and inflammatory response. BMC Complement Altern Med. 2016;16:360.

27. Ying $X$, Chen $X$, Cheng S, Shen $Y$, Peng $L, X u H Z$. Piperine inhibits IL- $\beta$ induced expression of inflammatory mediators in human osteoarthritis chondrocyte. Int Immunopharmacol. 2013;17(2):293-9.

28. Wu MY, Chao KH, Yang JH, Lee TH, Yang YS, Ho HN. Nitric oxide synthesis is increased in the endometrial tissue of women with endometriosis. Hum Reprod. 2003;18(12):2668-71.

29. Rocha MG, Gomes VA, Tanus-Santos JE, Rosa-e-Silva JC, Candido-dos-Reis FJ, Nogueira AA, et al. Reduction of blood nitric oxide levels is associated with clinical improvement of the chronic pelvic pain related to endometriosis. Braz J Med Biol Res. 2015;48(4):363-9.

30. Cheng W, Shivshankar P, Li Z, Chen L, Yeh IT, Zhong G. Caspase-1 contributes to Chlamydia trachomatis-induced upper urogenital tract inflammatory pathologies without affecting the course of infection. Infect Immun. 2008;76(2):515-22.

31. Hu XY, Wu RH, Logue M, Blondel C, Lai LYW, Stuart B, et al. Andrographis paniculata (Chuān Xīn Lián) for symptomatic relief of acute respiratory tract infections in adults and children: a systematic review and metaanalysis. PLOS ONE. 2017;12(8):e0181780.

32. Jayakumar T, Hsieh CY, Lee JJ, Sheu JR. Experimental and clinical pharmacology of Andrographis paniculata and its major bioactive phytoconstituent andrographolide. Evid Based Complement Altern Med. 2013;2013:846740.

33. Pholphana N, Rangkadilok N, Saehun J, Ritruechai S, Satayavivad J. Changes in the contents of four active diterpenoids at different growth stages in Andrographis paniculata (Burm.f.) Nees (Chuanxinlian). Chin Med. 2013;8(1):2.
34. Arifullah M, Namsa ND, Mandal M, Chiruvella KK, Vikrama P, Gopal GR. Evaluation of anti-bacterial and anti-oxidant potential of andrographolide and echiodinin isolated from callus culture of Andrographis paniculata Nees. Asian Pac J Trop Biomed. 2013;3(8):604-10.

35. Hua Z, Frohlich KM, Zhang Y, Feng X, Zhang J, Shen L. Andrographolide inhibits intracellular Chlamydia trachomatis multiplication and reduces secretion of proinflammatory mediators produced by human epithelial cells. Pathog Dis. 2015;73(1):1-11.

36. Shen T, Yang WS, Yi YS, Sung GH, Rhee MH, Poo H, et al. AP-1/IRF-3 targeted anti-inflammatory activity of andrographolide isolated from Andrographis paniculata. Evid Based Complement Altern Med. 2013;2013:210736.

37. Zou W, Xiao Z, Wen X, Luo J, Chen S, Cheng Z, et al. The anti-inflammatory effect of Andrographis paniculata (Burm. f.) Nees on pelvic inflammatory disease in rats through down-regulation of the NF-KB pathway. BMC Complement Altern Med. 2016;16(1):483.

38. Tao X, Sun X, Xu L, Yin L, Han X, Qi Y, et al. Total flavonoids from Rosa laevigata Michx fruit ameliorates hepatic ischemia/reperfusion injury through inhibition of oxidative stress and inflammation in rats. Nutrients. 2016;8(7):E418.

39. Zhao L, Xu L, Tao X, Han X, Yin L, Qi Y, Peng J. Protective effect of the total flavonoids from Rosa laevigata Michx fruit on renal ischemia-reperfusion injury through suppression of oxidative stress and inflammation. Molecules. 2016;21(7):E952.

40. Liu L, Chen L, Jiang C, Guo J, Xie Y, Kang L, et al. Berberine inhibits the LPS-induced proliferation and inflammatory response of stromal cells of adenomyosis tissues mediated by the LPS/TLR4 signaling pathway. Exp Ther Med. 2017;14(6):6125-30.

41. Ma X, Chen Z, Wang L, Wang G, Wang Z, Dong X, et al. The pathogenesis of diabetes mellitus by oxidative stress and inflammation: its inhibition by berberine. Front Pharmacol. 2018:9:782.

42. Jin H, Jin X, Cao B, Wang W. Berberine affects osteosarcoma via downregulating the caspase-1/IL-1 $\beta$ signaling axis. Oncol Rep. 2017;37(2):729-36.

43. Sweet RL. Treatment of acute pelvic inflammatory disease. Infect Dis Obstet Gynecol. 2011;2011:561909.

44. Wu YC, Hsieh CL. Pharmacological effects of Radix Angelica sinensis (Danggui) on cerebral infarction. Chin Med. 2011;6:32.

45. Chao WW, Lin BF. Bioactivities of major constituents isolated from Angelica sinensis (Danggui). Chin Med. 2011;6:29.

46. Liu C, Li J, Meng FY, Liang SX, Deng R, Li CK, et al. Polysaccharides from the root of Angelica sinensis promotes hematopoiesis and thrombopoiesis through the PI3K/AKT pathway. BMC Complement Altern Med. 2010;10:79

47. Huang Y, Chen L, Feng L, Guo F, Li Y. Characterization of total phenolic constituents from the stems of Spatholobus suberectus using LC-DADMS(n) and their inhibitory effect on human neutrophil elastase activity. Molecules. 2013;18(7):7549-56.

48. Fu YF, Jiang LH, Zhao WD, Xi-Nan M, Huang SQ, Yang J, et al. Immunomodulatory and antioxidant effects of total flavonoids of Spatholobus suberectus Dunn on PCV2 infected mice. Sci Rep. 2017;7(1):8676.

49. Yue $R$, Zhao $L$, Hu Y, Jiang $P$, Wang $S$, Xiang $L$, et al. Rapid-resolution liquid chromatography TOF-MS for urine metabolomic analysis of collagen-induced arthritis in rats and its applications. J Ethnopharmacol. 2013;145(2):465-75.

50. Ahn JK, Kim S, Hwang J, Kim J, Lee YS, Koh EM, et al. Metabolomic elucidation of the effects of curcumin on fibroblast-like synoviocytes in rheumatoid arthritis. PLOS ONE. 2015;10(12):e0145539.

51. Dai D, Gao Y, Chen J, Huang Y, Zhang Z, Xu F. Time-resolved metabolomics analysis of individual differences during the early stage of lipopolysaccharide-treated rats. Sci Rep. 2016;6:34136.

52. Qi Y, Jiang C, Cheng J, Krausz KW, Li T, Ferrell JM, et al. Bile acid signaling in lipid metabolism: metabolomic and lipidomic analysis of lipid and bile acid markers linked to anti-obesity and anti-diabetes in mice. Biochim Biophys Acta. 2015;1851(1):19-29.

53. Mariette C, Perrais M, Leteurtre E, Jonckheere N, Hémon B, Pigny $P$, et al. Transcriptional regulation of human mucin MUC4 by bile acids in oesophageal cancer cells is promoter-dependent and involves activation of the phosphatidylinositol 3-kinase signalling pathway. Biochem J. 2004;377(Pt 3):701-8.

54. Carraway KL, Ramsauer VP, Haq B, Carothers Carraway CA. Cell signaling through membrane mucins. BioEssays. 2003;25(1):66-71. 
55. Guijas C, Astudillo AM, Gil-de-Gómez L, Rubio JM, Balboa MA, Balsinde J. Phospholipid sources for adrenic acid mobilization in RAW 264.7 macrophages. Comparison with arachidonic acid. Biochim Biophys Acta. 2012;1821(11):1386-93.

56. Wu X, Cao H, Zhao L, Song J, She Y, Feng Y. Metabolomic analysis of glycerophospholipid signatures of inflammation treated with non-steroidal anti-inflammatory drugs-induced-RAW264.7 cells using (1)H NMR and U-HPLC/Q-TOF-MS. J Chromatogr B Analyt Technol Biomed Life Sci. 2016;1028:199-215

57. Dong L, Han $X$, Tao X, Xu L, Xu Y, Fang L, et al. Protection by the total flavonoids from Rosa laevigata Michx fruit against lipopolysaccharide-induced liver injury in mice via modulation of FXR signaling. Foods. 2018;7(6):E88.
58. Hu XY, Qin FY, Lu XF, Zhang LS, Cheng YX. Three new polyynes from Codonopsis pilosula and their activities on lipid metabolism. Molecules. 2018;23(4):E887.

59. Bröer $\mathrm{S}$, Bröer A. Amino acid homeostasis and signalling in mammalian cells and organisms. Biochem J. 2017;474(12):1935-63.

60. Zhenyukh O, González-Amor M, Rodrigues-Diez RR, Esteban V, RuizOrtega M, Salaices M, et al. Branched-chain amino acids promote endothelial dysfunction through increased reactive oxygen species generation and inflammation. J Cell Mol Med. 2018. https://doi.org/10.1111/ jcmm.13759.
Ready to submit your research? Choose BMC and benefit from:

- fast, convenient online submission

- thorough peer review by experienced researchers in your field

- rapid publication on acceptance

- support for research data, including large and complex data types

- gold Open Access which fosters wider collaboration and increased citations

- maximum visibility for your research: over 100M website views per year

At BMC, research is always in progress.

Learn more biomedcentral.com/submissions 